\title{
Ornithine transcarbamylase deficiency
}

INSERM

\section{Source}

INSERM. (1999). Orphanet: an online rare disease and orphan drug data base. Ornithine transcarbamylase deficiency. ORPHA:664

Ornithine transcarbamylase deficiency (OTCD) is a disorder of urea cycle metabolism and ammonia detoxification (see this term) characterized by either a severe, neonatal-onset disease found almost exclusively in males, or later-onset (partial) forms of the disease. Both present with episodes of hyperammonemia that can be fatal and which can lead to neurological complications. 